\title{
Isolierung von gelösten Kohlenhydraten aus dem Meerwasser
}

\author{
HeInZ SCHAEFeR ${ }^{1}$ \\ Biologische Anstalt Helgoland, Meeresstation, Helgoland
}

\begin{abstract}
Isolation of dissolved carbohydrates from sea water. The carbohydrates dissolved in sea water have been mostly determined in terms of total concentration. Only in a few cases have they been identified and single components measured quantitatively. In the present work we have studied the optimal conditions for the isolation of soluble sugars from sea water by means of carbon adsorption. One to 2 liters of sea water are passed through $13,8 \times 4 \mathrm{~cm}$ carbon-celite-columns, salts are washed out with $400 \mathrm{ml}$ of distilled water and sugars eluted with $400 \mathrm{ml}$ of $20 \%$ aethanol. The eluted sugar fraction is concentrated in vacuo, and the isolated sugars may be determined and identified by means of analytical micromethods. Water samples from several stations of the Deutsche Bucht (Southern North Sea) were collected, filtrated and analysed by the above mentioned method. Rhamnose, ribose, sucrose and one not yet identifed sugar were isolated. The carbon adsorption method might be of interest also for limnological studies.
\end{abstract}

\section{EINLEITUNG}

Die Gesamtmenge der gelösten Zucker im Meerwasser wird zur Zeit mit Anthron oder N-Athylcarbazol (Collier et al. 1953, Lewis \& Rakestraw 1955, Koyama 1962) bestimmt. Diese Methoden sind für Serienbestimmungen geeignet, da sie relativ einfach sind und direkt mit Seewasser durchgeführt werden können, ohne daß eine vorhergehende Isolierung der Zucker notwendig wäre. Jedoch wird die Genauigkeit der Anthronmethode durch die anwesenden Salze beeinträchtigt. Vergleicht man beide Methoden miteinander, dann erhält man unterschiedliche Werte (LewIS \& RAKESTRAW 1955). Die Gesamtmenge der Kohlenhydrate wird meist als Saccharose (Sucrose) angegeben, da die einzelnen Zuckerarten unterschiedlich angefärbt werden (vgl. HoppeSeyler \& Thierfelder 1955, p. 694): $100 \gamma$ Glucose sind bei der Anthronmethode farbäquivalent $100 \gamma$ Fructose, $185 \gamma$ Galaktose, $95 \gamma$ Maltose, $130 \gamma$ Lactosehydrat, $90 \gamma$ Glycogen. Falls in den zu untersuchenden Meerwasserproben unterschiedliche Mengen an verschiedenen Zuckerarten vorkommen - und das dürte in der Regel der Fall sein - kann die Bestimmung der Gesamtkohlenhydrate mit einem sehr großen Fehler belastet sein; die tatsächlichen Verhältnisse werden daher zumindest häufig nicht wiedergegeben. In dieser Hinsicht soll die von ZEIN-ELDIN \& MAY (1958) verbesserte Methode mit N-Äthylcarbazol exakter sein, da Pentosen, Hexosen und Polymere mit Ausnahme von Xylose und Fructose dieselbe Färbungsintensität ergeben. \footnotetext{
$43-45$.

1 Der Deutschen Forschungsgemeinschaft danke ich für die Unterstïtzung der Arbeit.

Neue Anschrift des Verfassers: Dr. H. Schaefer, 673 Neustadt/Weinstr., Maximilianstr.
} 
In vielen Fällen aber ist es wünschenswert zu wissen, welche Kohlenhydrate im Meerwasser und in marinen Planktonkulturen vorkommen, und wie hoch die Konzentration der einzelnen Zucker ist. Auf ihre Isolierung kann dann nicht verzichtet werden. Für diesen $Z$ weck wurden bisher mehrere Verfahren diskutiert beziehungsweise angewandt. Das Verfahren von Degens et al. (1964) (Extraktion von eingedampttem Seewasser, anschließend Restentsalzung durch Ionenaustauscher) wurde bereits in einer vorhergehenden Arbeit (Schaefer 1965) diskutiert. Jefreey \& Hood (1958) haben verschiedene andere Methoden überprüf. Zur Isolierung der Kohlenhydrate eignete sich am besten die Adsorption an Aktivkohle. WANGERSKY (1952) hatte schon früher mit dieser Methode Ascorbinsäure und ein Rhamnosid aus Seewasser isolieren können. JoHnston (1955) schlug vor, Seewasser mit Aktivkohle zu schütteln und die Kohle nach dem Freiwaschen von Salzen zu extrahieren. Dieses Vorgehen hat sich in meinem Laboratorium nicht bewährt, da dabei starke Verluste an Kohlenhydraten (etwa 60\%), vermutlich bedingt durch den Auswascheffekt, auftraten. Unter Einhaltung bestimmter Bedingungen ist die Verwendung von Ionenaustauschern zwar auch geeignet, doch ist das Verfahren für Meerwasser sehr aufwendig.

$\mathrm{Da}$ in meinem Laboratorium weder die Kopräzipitationsmethode von JEFFrEY \& Hoop noch die Extraktionsmethode von DEgens et al. befriedigende Ergebnisse lieferte, überprüfte ich das Ionenverzögerungsverfahren nach HATcH et al. (1957). Die Vorversuche verliefen sehr günstig (SCHAEFER 1964). Desgleichen zeigten Versuche mit 11 Meerwasserproben, daß sich das Verfahren in der Praxis bewährte (Schaefer 1965). Aus dem Kulturmedium von Artemia salina und einer Grünalge konnten Rhamnose und zwei weitere Zucker isoliert werden. Jedoch zwangen mich gewisse Schwierigkeiten, die sich mit neuerdings hergestellten Chargen von Retardion $11 \mathrm{~A} 8 \mathrm{8}$ ergaben, nach einem weiteren und auch einfacheren Verfahren zu suchen. Es wurde daher auf die Adsorptionsmethode mit Aktivkohle zurückgegriffen und nach optimalen Versuchsbedingungen gesucht. Diese Bedingungen sowie eine Arbeitsvorschrift und einige Anwendungsbeispiele werden im folgenden dargelegt.

\section{METHODIK}

Nach Whrstrer \& Durso (1950) werden Kohlenhydrate an Säulen, die Aktivkohle und Celite im Verhältnis $1: 1$ enthalten, mehr oder weniger stark adsorbiert. Monosaccharide lassen sich mit größeren Wassermengen, Di- und Oligosaccharide aber erst mit verdünnten alkoholischen Lösungen eluieren. Anorganische Salze passieren die Säule. Um optimale Versuchsbedingungen zu erhalten, mußten mehrere Faktoren ermittelt werden: (1) Durchlaufgeschwindigkeit, (2) Säulengröße und Bettvolumen für 1 oder mehrere Liter Meerwasser, (3) benötigte Wassermenge, um die Meerwassersalze auszuwaschen, (4) Feststellung des Elutionsvolumens für die Zucker, (5) Ausbeute zugesetzter 'Testsubstanzen.

Für die Versuche wurden im allgemeinen Säulen mit den Abmessungen $30 \times 4 \mathrm{~cm}$, Betthöhe Aktivkohle-Celite-Mischung $13,8 \mathrm{~cm}$ (etwa den Angaben von JEFrREY \& HOoD entsprechend) verwendet, die am unteren Ende mit einer eingeschmolzenen $G 3$ (Schott-)Glasfritte versehen waren. Ohne Zugabe von Celite ist die Durchlaufgeschwin- 
digkeit äußerst gering. Mit obigen Säulen konnten Durchlaufgeschwindigkeiten von 150 bis $200 \mathrm{ml} / \mathrm{h}$ erzielt werden. Bei Veränderung des Mischungsverhältnisses KohleCelite von $1: 1$ auf $1: 2$ kann die Durchlaufgeschwindigkeit noch etwas erhöht werden. Ist diese aber zu hoch, dann werden die Kohlenhydrate nicht mehr quantitativ adsorbiert. So wurden beispielsweise bei $750 \mathrm{ml} / \mathrm{h}$ und 500 Torr nur noch etwa $82 \%$ der zugesetzten Glucose wiedergefunden. 150 bis $200 \mathrm{ml} / \mathrm{h}$ erscheinen arbeitsmäßig als optimal, da eine 1 bis 21 Wasserprobe dann über Nacht die Säulen durchlaufen kann. Die Durchlaufgeschwindigkeit ist bei Säulen mit geringerem Durchmesser oder größerer Betthöhe herabgesetzt.

Zur Identifizierung der einzelnen Kohlenhydrate muß das Eluat salzfrei sein. Es wäre also optimal, wenn beim Auswaschen der Salze aus der Säule noch keine Zucker im wäßrigen Eluat erscheinen würden. Vorversuche ergaben, daß zum Auswaschen der Salze etwa $400 \mathrm{ml}$ aqua destillata benötigt werden, danach sind nur noch Spuren von Chloriden und Sulfaten nachweisbar. Glucose erscheint noch nicht in dieser Fraktion. Damit ist eine saubere Trennung von Zucker und anorganischen Salzen gesichert.

Da einerseits für das Auswaschen der Monosaccharide größere Wassermengen benötigt werden, andererseits in den Isolierungsversuchen auch $\mathrm{Di}$ - und Trisaccharide erfaßt werden sollen, die sich nicht mit Wasser, sondern mit verdünntem Alkohol eluieren lassen, wurden die Kohlenhydrate in den weiteren Versuchen nur mit 20\% Athanol desorbiert. Nach WhIstler \& Durso (1950) besteht zwischen Elutionsvolumen und Alkoholkonzentration in den niedrigen Konzentrationsstufen eine gewisse Beziehung derart, daß zum Beispiel das Elutionsvolumen bei 15\% Alkohol um etwa das Dreifache herabgesetzt wird gegenüber 10\% Alkohol. Bei den angegebenen Säulenabmessungen erscheinen Arabinose, Glucose und Raffinose zwischen 120 und $300 \mathrm{ml}$ Durchfluß nach Aufgabe von 20\% Äthanol.

Um die Zahl der Arbeitsschritte auf ein Minimum zu beschränken, wurden bei den Ausbeuteuntersuchungen $400 \mathrm{ml}$ Eluat, beginnend mit der Aufgabe von $400 \mathrm{ml} \mathrm{20 \%}$ Äthanol auf die Säule, gesammelt. Damit müßten Pentosen bis Trisaccharide quantitativ im Eluat erscheinen. Höhere Zucker wurden nicht untersucht.

Da bei Zugabe von Athanol ein gewisses Zerreißen der Säulenfüllung entsteht, empfiehlt es sich, die Durchlaufgeschwindigkeit beim Eluieren auf etwa 80 bis $100 \mathrm{ml} / \mathrm{h}$ zu drosseln.

Ausbeuteuntersuchungen ergaben, daß bei obiger Durchflußgeschwindigkeit in 11 Meerwasser gelöste Arabinose, Xylose, Glucose, Saccharose, Raffinose, Sorbit und Mannit zwischen 98 und 101\% im Eluat von $400 \mathrm{ml} 20 \%$ Athanol wiedergefunden wurden. Die Schwankung der Ergebnisse liegt innerhalb der Fehlergrenze der angewandten Nachweismethoden (Nelson- bzw. Perjodatmethode). Verluste von Zuckern treten also nicht auf.

In dem Zuckereluat wurden durchschnittlich nur noch $6 \mathrm{mg}$ Chlorid und geringste Spuren von Sulfat gefunden. Die Lösung ist also praktisch salzfrei.

Mit den angegebenen Säulen lassen sich die Monosaccharide auch aus 21 Meerwasser zu $100 \%$ isolieren. Ab 31 pro Probe werden die Zucker nicht mehr quantitativ wiedergefunden. 


\section{ARBEITSVORSCHRIFT}

$100 \mathrm{~g}$ Aktivkohle (Merck) und $100 \mathrm{~g}$ Celite (Merck) werden in Wasser suspendiert und gründlich vermischt. Nach Absitzen wird dekantiert. Das Gemisch wird mit der zehnfachen Menge 20\% Essigsäure einige Minuten lang gekocht, heiß abgesaugt und mit heißem Wasser 2- bis 3 mal nachgewaschen (vgl. PAECH \& Tracey 1956, p. 106). Die Mischung wird dann in eine Säule 30 bis $40 \times 4 \mathrm{~cm}$ eingeschlämmt, und zwar so viel, daß nach dem Absitzen eine Betthöhe von 13 bis $14 \mathrm{~cm}$ verbleibt. Die Oberfläche wird mit einigen Lagen Filtrierpapier bedeckt, um ein Aufwirbeln bei der Zugabe der Analysenproben zu vermeiden. Nach dem Durchlauf von $30 \mathrm{ml}$ Pufferlösung $(0,2 \mathrm{~m}$ Citratpuffer, pH 7) zum Entfernen von Eisenspuren (PAECH \& TRACEY 1956, p. 106) und Nachwaschen mit aqua destillata ist die Säule gebrauchsfertig.

Bis zu 21 Analysenprobe werden vorsichtig auf die gerade noch feuchte Kohlenoberfläche gegeben. Nach Einsickern der Probe wird mit $400 \mathrm{ml}$ aqua destillata und

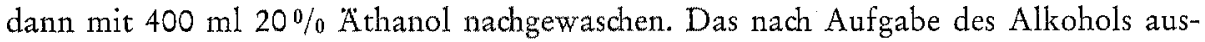
laufende Eluat wird aufgefangen, im Vakuum auf ein gewünschtes Volumen eingedampft, eventuell gefriergetrocknet und analysiert. Die Elution mit Alkohol verläuft bei weniger als $100 \mathrm{ml} / \mathrm{h}$. Zum späteren Auswaschen des Alkohols aus der Säule werden mindestens $200 \mathrm{ml}$ aqua destillata benötigt. Die Säule ist dann wieder einsatzfähig.

Wenn die Durchlaufgeschwindigkeit im Verlaufe etlicher Versuche geringer wird oder wenn starke Risse auftreten, entfernt man die Säulenfüllung, rührt tüchtig und schlämmt wieder ein. Statt dessen ist auch ein Zurückwaschen möglich, wobei man die Säulenfüllung von unten her mit Wasser hochdrückt und dann sedimentieren läßt.

Die oben aufgefuhrte Begrenzung des Probevolumens auf 21 gilt nur für den Fall, daß auch Monosaccharide isoliert werden sollen. Da Di- und Trisaccharide nicht mit Wasser desorbiert werden, dürfte zur alleinigen Isolierung dieser Substanzen aus dem Meerwasser die Behandlung einer noch größeren Analysenprobe möglich sein.

Falls das Meerwasser Gelbstoffe enthält, ist es angebracht, die Wasserprobe zuerst durch eine kleine Säule mit wenigen $\mathrm{cm}$ Betthöhe von Retardion 11A8 und dann erst durch die Kohlensäule zu leiten, da Retardion einen Teil der Gelbstoffe adsorbiert (SCHAEFER 1964).

Sollen größere Mengen Seewassers analysiert werden, verwende man entsprechend mehr Kohlesäulen obiger Abmessungen, da die sonst notwendige Vergrößerung der Betthöhe einer einzigen Säule zu einer geringeren Durchflußgeschwindigkeit führt.

Da die Adsorptionsmethode sehr einfach und billig ist, lassen sich bequem Reihenanalysen mit einer entsprechenden Säulenzahl durchführen. Mit dem Verfahren kann auch an Bord eines Schiffes gearbeitet werden, wenn dafür Sorge getragen wird, daß die Oberfläche der Säulenfüllung nicht erschüttert wird. Zu diesem $\mathrm{Zwe}$ we bringt man auf die auf der Kohle liegende Filterschicht einen Kunststoffeinsatz, der genau in die Säule hineinpaßt und unten eine großporige Filterplatte besitzt. 


\section{ANWENDUNGSBEISPIELE}

An mehreren Stellen der Deutschen Bucht wurden Wasserproben entnommen, in Plastikflaschen eingefüllt und spätestens nach 8 Stunden eingefroren oder, im Fall der Kabeltonne (zwischen Helgoland und Düne), nach spätestens einer halben Stunde filtriert und verarbeitet. Die Proben wurden zunächst durch ein Perlonfilter und dann durch ein Membranfilter (Porenweite $0,25 \mu$ ) bei 400 bis 500 Torr filtriert und auf die Säule gegeben. Das alkoholische Eluat wurde im Rotationsverdampfer fast bis zur Trodkne eingeengt, gefriergetrocknet, mit 0,1 bis $0,5 \mathrm{ml}$ aqua destillata aufgenommen und eindimensional papierchromatographisch unter Mitlaufen von Vergleichssubstanzen untersucht. Als Laufmittel dienten n-Butanol-Eisessig-Wasser 4: $1: 1$ und ButanolPyridin-Wasser 6:4:3. Die Anfärbung erfolgte mir Triphenyltetrazoliumchlorid und mit Perjodat-Benzidin- $\mathrm{KMnO}_{4}$ (HaIs \& MaceK 1958, D 28b). Die Ergebnisse einiger Anwendungsbeispiele bringt Tabelle 1.

\section{Tabelle 1}

Gelöste Kohlenhydrate in Meerwasserproben aus der Deutschen Bucht. 11 Wasserproben; $0,5 \mathrm{~m}$ Tiefe

\begin{tabular}{|c|c|c|c|}
\hline Station & Datum & Position & gefundene Zucker \\
\hline \multirow{3}{*}{$\begin{array}{l}\text { Kabeltonne } \\
\text { (zwischen Helgo- } \\
\text { land und Düne) }\end{array}$} & 30. 9.64 & $\begin{array}{l}54011,3^{\prime} \mathrm{N} \\
7053,9^{\prime} \mathrm{E}\end{array}$ & 1 nicht identifizierter Zucker \\
\hline & 2. 10.64 & & $\begin{array}{l}\text { Rhamnose (etwa } 500 \mathrm{\gamma} / \mathrm{l} \text { ) } \\
1 \text { nicht identifizierter Zucker, wie oben }\end{array}$ \\
\hline & 6.11 .64 & & $\begin{array}{l}\text { Rhamnose (etwa } 500 \gamma / 1 \text { ) } \\
\text { Ribose (Spur) } \\
\text { Saccharose (etwa } 100 \gamma / 1 \text { ) }\end{array}$ \\
\hline Helgolandtonne & 20.1 .65 & $\begin{array}{l}54^{0} 9,1^{\prime} \mathrm{N} \\
7^{0} 53,5^{\prime} \mathrm{E}\end{array}$ & $\begin{array}{l}\text { Rhamnose }(300-400 y / 1) \\
\text { Saccharose (etwa } 100 \mathrm{y} / 1) \\
1 \text { nicht identifizierter Zucker, wie oben } \\
\end{array}$ \\
\hline Tonne H 1 & 20. 1.65 & $\begin{array}{ll}54^{\circ} & 3,0^{\prime} \mathrm{N} \\
8^{0} & 5,0^{\prime} \mathrm{E}\end{array}$ & $\begin{array}{l}\text { Rhamnose (etwa } 500 \gamma / 1 \text { ) } \\
\text { Saccharose (etwa } 100 \mathrm{y} / 1 \text { ) } \\
1 \text { nicht identifizierter Zucker, wie oben }\end{array}$ \\
\hline Tonne B & 20. 1.65 & $\begin{array}{r}53059,4^{\prime} \mathrm{N} \\
8018,7^{\prime} \mathrm{E} \\
\end{array}$ & Rhamnose (etwa $300-400 \mathrm{\gamma} / \mathrm{l}$ ) \\
\hline Tonne Mittelhever & 21. 1.65 & $\begin{array}{l}54^{0} 22,0^{\prime} \mathrm{N} \\
8^{0} 17,5^{\prime} \mathrm{E}\end{array}$ & $\begin{array}{l}\text { Rhamnose (etwa } 500 \gamma / 1 \text { ) } \\
1 \text { nicht identifizierter Zucker, wie oben }\end{array}$ \\
\hline
\end{tabular}

\section{DISKUSSION}

Wie eingangs erwähnt wurde, hat man bisher mehrmals die Gesamtkohlenhydrate im Meerwasser bestimmt, wobei zum Teil auch Vertikalschnitte durchgeführt worden sind. Lewis \& Rarestraw (1955) fanden bis zu 0,45 mg Zucker (als Sucrose ausge- 
drückt) in 11 Seewasser von der kalifornischen Küste, in Lagunen sogar bis zu 5,3 mg/l. Nach Koyama (1962) wurden im Nordwestpazifik bis zu $1,68 \mathrm{mg} / \mathrm{l}$, von Collier (1953) bis zu $2 \mathrm{mg} / 1$ in Proben aus dem Golf von Mexiko gefunden. Es ist jedoch noch nicht eindeutig festgestellt worden, ob es sich hier auch wirklich nur um Kohlenhydrate handelte. Collner wies bereits 1953 darauf hin, daß man hier besser nicht von Kohlenhydraten, sondern von N-Äthylcarbazol-(positiven-) Substanzen sprechen sollte. Dieses Faktum allein zeigt schon, wie wichtig es ist, nicht summarische Gruppenanalysen durchzuführen, sondern die einzelnen Komponenten zu identifzieren und diese quantitativ zu bestimmen. Lediglich in diesem Fall verfügen wir über eindeutige Angaben, wie sie für die Beurteilung des Stoffhaushaltes im Meer erforderlich sind. Nur auf diese Weise können wir Kenntnisse über die Herkunft der gelösten organischen Substanzen des Meerwassers erlangen. Daraus ergibt sich die Notwendigkeit der Ausarbeitung von Isolierungsverfahren, von denen eines in der vorliegenden Arbeit ausführlich beschrieben wurde.

Die Identifizierung von gelösten Kohlenhydraten aus dem Meerwasser ist jedoch bisher nur selten durchgeführt worden. WANGERSKY (1952) konnte ein Rhamnosid sowie Ascorbinsäure feststellen. Collrer (1953) fand 1952 während der „red tide" bei Florida bis zu $50 \mathrm{mg} / \mathrm{l}$ Rhamnosid. Degens et al. (1964) identifizierten Galaktose, Glucose, Mannose, Rhamnose und Arabinose in kalifornischen Gewässern, wobei sie die Konzentrationen der einzelnen Zuckerarten quantitativ bestimmten. Deren Mengen nahmen zu mit der Wassertiefe. In $3890 \mathrm{~m}$ Tiefe fand er insgesamt 36,3 $\gamma / 1$ Hexosen und Pentosen.

In der vorliegenden Arbeit wurden nunmehr auch für die Deutsche Bucht Kohlenhydrate nachgewiesen. Aus den Papierchromatogrammen ist zu schließen, daß Rhamnose in höheren Konzentrationen vorkam als die übrigen Zucker. Überdies wurde Rhamnose fast in jeder Probe gefunden. Auf Grund der bisher noch kargen Befunde erübrigt sich eine eingehendere Diskussion; es ist jedoch bekannt, daß Phytoplankton und Algen eine große Zahl von verschiedenen Kohlenhydraten enthalten (McKINNEL \& Percival 1962, Marshall \& Orr 1962, und andere), wobei Rhamnose unter anderem ein wichtiger Bestandteil eines Polysaccharids der Alge Enteromorpha ist.

Da die vorliegende Arbeit abgebrochen werden mußte, war es nicht mehr möglich, exakte quantitative Bestimmungen der gefundenen Zucker durchzuführen und den noch unbekannten Zucker zu identifizieren. Die in der Tabelle 1 angegebenen Konzentrationen sind größenordnungsmäßige Schätzungen auf Grund der bekannten Menge der mitgelaufenen Vergleichssubstanzen.

Das geschilderte Verfahren dürfte auch für die Isolierung von Kohlenhydraten aus dem Süßwasser geeignet sein. Hier können mit einer Kohlensäule große Wassermengen verarbeitet werden, falls deren Volumen vor der Aufgabe auf die Säule auf 1 bis 21 reduziert wird.

\section{ZUSAMMENFASSUNG}

1. Der Wert der summarischen Bestimmung der Gesamtmenge von im Meerwasser gelösten Kohlenhydraten wird diskutiert. 
2. Die optimalen Versuchsbedingungen für die Isolierung von gelösten Kohlenhydraten aus 1 bis 21 Meerwasser mit Hilfe des Kohleadsorptionsverfahrens werden mitgeteilt. In dem salzfreien Eluat der Kohle-Celite-Säulen können die einzelnen Zucker mit Mikronachweisverfahren quantitativ analysiert werden.

3. Aus Seewasserproben (Deutsche Bucht) wurden mit der geschilderten Methode Rhamnose, Ribose, Saccharose und ein noch nicht identifizierter Zucker isoliert.

Dem Direktor der Biologischen Anstalt Helgoland, Herrn Prof. Dr. O. KInNe, danke ich für einen Arbeitsplatz und für sein großes Interesse an der vorliegenden Arbeit. Besonders danke ich Herm Dr. H. Aurich, Litoralstation der Biologischen Anstalt Helgoland (List auf Sylt) für seine Unterstützung. Herr Dr. E. HAGMEIER, Meeresstation der BAH., besorgte mir liebenswürdigerweise die Wasserproben.

\section{ZITIERTE LITERATUR}

Collier, A., 1953. The significance of organic compounds in sea water. Trans. N. Am. Wildl. Conf. 18, 463-472.

Collier, A., Ray, S., Magnitzky, A. W. \& Bell, J. O., 1953. Effect of dissolved organic substances on oysters. Fishery Bull. Fish Wildl. Serv. U. S. 54, 167-185.

Degens, E. T., Reuter, J. H. \& Shaw, K. N. F., 1964. Biochemical compounds in offshore California sediments and sea waters. Geodhim. cosmochim. Acta 28, 45-66.

HaIs, I. M. \& MACEK, K., 1958. Handbuch der Papierchromatographie. A. d. Tschech. Bd. 1. G. Fischer, Jena, 860 pp.

Hatch, M. J., Dillon, J. A. \& SMrth, H. B., 1957. Preparation and use of snake-cage poly electrolytes. Ind. Engng. Chem. 49, 1812-1819.

HopPe-Seyler, G. \& THIerfelder, H., 1955. Handbuch der physiologisch- und pathologischchemischen Analyse. 10. Aufl. Bd 3. T. 1. 2. Springer, Berlin.

JefFrey, L. M. \& Hood, D. W., 1958. Organic matter in sea water; an evaluation of various methods for isolation. J. mar. Res. 17, 247-271.

Johnston, R., 1955. Biologically active compounds in the sea. J. mar. biol. Ass. U. K. 34, 185-195.

Koyama, T., 1962. Organic compounds in sea water. J. oceanogr. Soc. Japan 20, 563-576.

Lewis, G. J. \& Rakestraw, N. W., 1955. Carbohydrate in sea water. J. mar. Res. 14, 253-258.

Marshall, S. M. \& ORR, A. P., 1962. Carbohydrate as a measure of phytoplankton. J. mar. biol. Ass. U. K. 42, 511-519.

McKinnell, J. P. \& Percrval, E., 1962. Structural investigations on the water-soluble polysaccharide of the green seaweed Enteromorpha compressa. J. chem. Soc. 1962, 3141-3148.

PaECh, K. \& Tracey, M. V., 1956. Moderne Methoden der Pflanzenanalyse. Bd. 1. Springer, Berlin, 542 pp.

SChAEfer, H,, 1964. Beiträge zur Entsalzung mit Retardion 11A8. Helgoländer wiss. Meeresunters. 11, 301-322.

- 1965. Isolierung von gelösten organischen Verbindungen aus dem Meerwasser unter besonderer Berücksichtigung der Aminosäuren. Helgoländer wiss. Meeresunters. 12, 239-252.

WANGERSKY, P. J., 1952. Isolation of ascorbic acid and rhamnosides from sea water. Science, N. $Y .115,685$.

Whistler, R. L \& Durso, D. F., 1950. Chromatographic separation of sugars on charcoal. J. Am. chem. Soc. 72, 677-679.

ZeIN-ELDIN, Z. P. \& MAY, B. Z., 1958. Improved N-ethylcarbazole determination of carbohydrates with emphasis on sea water samples. Analyt. Chem. 30, 1935-1941. 